\title{
Effect of Confinement Layer on Laser Ablation and Cavitation Bubble during Laser Shock Peening
}

\author{
Tomoki Takata ${ }^{1, *}$, Manabu Enoki $^{1}$, Pornthep Chivavibul $^{1}$, Akinori Matsui ${ }^{2}$ and Yuji Kobayashi ${ }^{2}$ \\ ${ }^{1}$ Department of Materials Engineering, The University of Tokyo, Tokyo 113-8656, Japan \\ ${ }^{2}$ SHINTOKOGIO, LTD., Nagoya 460-0003, Japan
}

Laser shock peening (LSP) is one of surface treatments to induce residual compressive stresses near metal surface to improve the resistance of materials to surface-related failures, such as fatigue and stress corrosion cracking. In LSP process, short pulsed laser is focused and irradiated to the material covered by transparent overlay such as glass or water. This transparent overlay is also known as a confinement layer and has an important role to increase impact pressure during LSP process. When confinement layer is liquid, the characteristics of the layer such as temperature, viscosity, etc. affect the phenomena occurring during the peening process and undoubtedly influence the induced residual stress. In the present study, Acoustic Emission (AE) technique coupling with high speed camera was applied to study the effect of confinement layer on LSP process. The results were discussed using the impact force calculated by inverse analysis of detected AE waveforms and bubble parameters observed by high speed camera. The results showed that temperature, thickness and viscosity of confinement layer had strong influence on the generation and collapse of cavitation bubble. The optimization of process parameters could be obtained by AE technique.

[doi:10.2320/matertrans.M2016150]

(Received April 28, 2016; Accepted July 29, 2016; Published September 25, 2016)

Keywords: laser shock peening, acoustic emission method, inverse analysis, cavitation bubble

\section{Introduction}

Peening is one of surface treatment methods to improve the fatigue strength of metals by inducing residual compressive stresses generated from mechanical means such as abrasive blasting, which is called shot peening. Among various peening techniques, laser shock peening (LSP) is a surface treatment technique, which uses laser for material processing instead of abrasives in conventional shot peening. LSP is superior to shot peening in views of the maximum values and the depth of residual compressive stress ${ }^{1)}$. Shock wave by laser irradiation makes material surface deform plastically and residual compressive stress generates at plastic deformed area because of restriction by undeformed area. Since an area treated by one pulse is small, laser has to be irradiated many times with moving laser or material for surface treatment. However, this technique provides many advantages such as non-contact, possibility to process on desired areas. LSP is applied to steam turbines, aerospace field and nuclear vessel for improving the fatigue properties of various metals ${ }^{2)}$.

The laser can be directly irradiated onto a bare metal surface or via a transparent overlay covered on the surface in LSP process. In the latter case, the overlay, which is also known as the confinement layer, confines the plasma from laser ablation and then the laser energy concentrates into narrow area. Such phenomena result in an increase of the pressure by plasma up to several GPa, which is several times higher than that obtained without the confinement layer. There are several kinds of confinement layer such as water, quartz, glass, silicon rubber ${ }^{3,4)}$. The choice of confinement layer depends on the substrate material, optical transparency for laser wavelength and acoustic impedance, which depends on density and acoustic velocity of confinement layer and affects the pressure by laser irradiation ${ }^{5)}$. Water is often chosen as the higher threshold for dielectric confinement material because it is much easier to apply than solid materials. Moreover, breakdown rarely generates in water compared to crystalline materials, therefore an application of laser pulses with higher intensities for peening is available ${ }^{6)}$. The properties of confinement layer of LSP have a strong influence on the peening quality. Therefore, it is important to investigate the effect of confinement layer on LSP.

The residual stress in depth direction is generally used to evaluate the degree of LSP. There are destructive and non-destructive methods to get residual stress data. A sample for the first method has to be cut and polished before the test. The latter ones include X-ray diffraction ${ }^{7)}$ and VISAR methods ${ }^{8)}$. There are still some limitations for these methods for applying to industrial process. For example, X-ray diffusion measurement has to be conducted after peening process. VISAR method can be only applied to thin samples. Acoustic emission (AE) method is one of the nondestructive evaluation methods to evaluate the size, location and generation time of material deformation and damage. This method is usually applied as an online monitoring for many industries. This method was successfully applied to both conventional shot peening $^{9)}$ and $\mathrm{LSP}^{10)}$ to evaluate the impact force by inverse analysis of detected waveform.

In the present study, AE technique was applied with high speed camera to investigate the phenomena during LSP. The effect of confinement layer was focused by varying its temperature, layer thickness and viscosity. Water was selected as a main confinement layer to study the effect of temperature and layer thickness while the effect of viscosity was studied by varying a mixture of water and glycerin. The results obtained from AE measurement was systematically related to those obtained from high speed camera to explain the effect of each confinement layer parameter on LSP process. 


\section{Experimental Methods}

\subsection{Material}

An aluminum alloy A7075, which is used in aerospace field, was used as a sample in the present study. This alloy was cut into a dimension of $50 \times 50 \times 10 \mathrm{~mm}$ and one of the $50 \times 50 \mathrm{~mm}$ surfaces was polished and cleaned in acetone before the test. The specimen was fixed by a jig in the tank. This jig provided a possibility to move the specimen to a desired location in three axis directions.

\subsection{Laser shock peening and high speed camera}

A Q switched Nd: YAG laser (Tempest 20, New Wave Research), which has a wavelength of $532 \mathrm{~nm}$, a pulse width of 3-5 ns and a maximum output power of $100 \mathrm{~mJ}$ was used for LSP. The laser was focused onto the center of the polished surface of the sample by a lens. The diameter of the laser beam on the sample surface was approximately $0.7 \mathrm{~mm}$. Two experimental setups were arranged to investigate the effects of confinement layer. Figure 1(a) shows a schematic diagram of the experiment setup to evaluate the effect of water temperature on LSP. The sample was set in a thermostat tank and the laser was irradiated to sample surface passing through the lens and acrylic tank wall in the horizontal direction. The sample was set 60-mm apart from the lens instead of a distance equal to focus length of the lens due to a difference in refractive index between air and water. The water temperature was varied from 278 to $323 \mathrm{~K}$. Figure 1(b) shows a schematic diagram of the experiment setup to evaluate the effect of water thickness on LSP. The sample was placed in the horizontal direction, therefore, the water thickness was easily varied from 1 to $10 \mathrm{~mm}$. The water temperature was kept constant at $295 \mathrm{~K}$.

The same experiment setup as shown in Fig. 1(b) was used
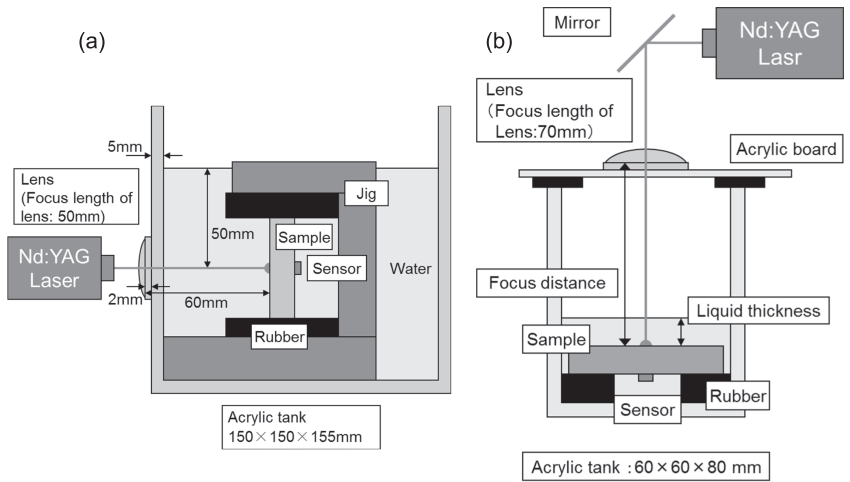

Fig. 1 (a) Experimental setup 1 and (b) experimental setup 2 of laser shock peening.

Table 1 The density and viscosity of the confinement layer of each glycerin percentage.

\begin{tabular}{ccc}
\hline $\begin{array}{c}\text { Glycerin percentage } \\
/ \text { mass } \%\end{array}$ & Density $/ \mathrm{g}^{\mathrm{cm}} \mathrm{cm}^{-3}$ & $\begin{array}{c}\text { Dynamic viscosity } \\
/ 10^{-3} \mathrm{~Pa} \cdot \mathrm{s}\end{array}$ \\
\hline 0 & 0.998 & 1.005 \\
24 & 1.06 & 2.118 \\
65 & 1.17 & 12.33 \\
83 & 1.22 & 123.6 \\
100 & 1.26 & 1410 \\
\hline
\end{tabular}

to evaluate the effect of viscosity of confinement layer. The thickness and temperature of confinement layer were kept constant at $10 \mathrm{~mm}$ and $295 \mathrm{~K}$, respectively. The various viscosities of confinement layer were obtained by mixing glycerin $(0,24,65,83$ and 100 mass $\%)$ with pure water. Table 1 shows the viscosity of confinement layer obtained from the calculation of literature data ${ }^{11)}$.

A high speed camera (Phantom Miro M110, Vision Research ${ }^{\circledR}$ Inc.) was used mainly to observe phenomena happend at the sample surface for LSP tests. This camera provided a maximum photograph speed of $220000 \mathrm{fps}$ with a resolution of $64 \times 64$ pixels.

\subsection{AE measurement and inverse analysis}

An AE sensor (PICO, Physical Acoustics Corp.) was attached at the center of the rear surface to detect elastic wave generated during LSP as shown in Figs. 1(a) and (b). This sensor has operating frequency range of $500-1850 \mathrm{kHz}$ and a resonant frequency of $600 \mathrm{kHz}$. Since the sensor was soaked in the tank, the cable of the sensor was covered by waterproof sealant paste. AE waveforms was recorded by using Continuous Wave Memory $(\mathrm{CWM})^{12)}$, which was been developed by our research group and is capability to memorize all waveforms continuously at the sampling rate of $10 \mathrm{MHz}$. The times of both AE measurement and high speed camera were synchronized providing a correct relationship of phenomena observed by high speed camera with detected AE waveforms.

An inverse analysis was applied to detected AE waveform to evaluate the impact force generated during LSP. Detected AE waveform, $V(t)$ can be represented following equation:

$$
V(t)=S(t) * G(t) * I(t)
$$

where, * represents convolution technique, and $S(t), G(t)$ and $I(t)$ are response function of sensor, Green's function of sample and source function of impact force, respectively. $S(t)$ and $G(t)$ could be obtained from a simulation of AE waveform by finite element method and experimental method of sharp pencil lead breaking ${ }^{13)}$. Then, $I(t)$ was estimated by the deconvolution technique from detected $V(t)$, calculated $S(t)$ and $G(t)^{14)}$.

\section{Results and Discussion}

\subsection{Example of AE waveforms and observation by high speed camera during LSP}

Figure 2 shows example of AE waveforms detected during LSP process in experimental setup as shown in Fig. 1(b) op-

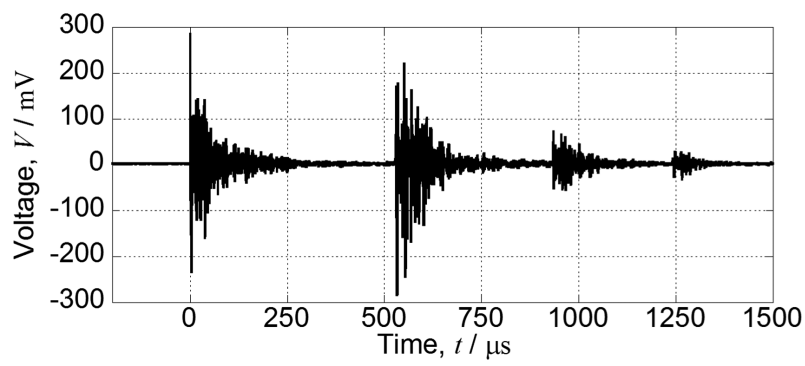

Fig. 2 Example of detected AE waveform during laser shock peening at laser power density of $5.0 \mathrm{GW} / \mathrm{cm}^{2}$. 
erated at water thickness of $5 \mathrm{~mm}$ and laser power density of $5.0 \mathrm{GW} / \mathrm{cm}^{2}$. For a shot of laser irradiation, several AEs could be detected with a time lag of several hundred microseconds. The arrival times of the first to forth AE waveforms were approximately 0, 530, 935 and $1242 \mu$ s, respectively, therefore the time intervals between adjacent AEs were approximately 530, 405 and $307 \mu$ s, respectively. Figure 3 shows sequential images recorded by the high speed camera during the same experiment of AE waveform shown in Fig. 2. According to the high speed camera observation, after laser ablation (Fig. 3(a)), a bubble generated (Fig. 3(b)) and expanded its size up to the maximum radius (Fig. 3(c) and (d)). Then, the bubble became smaller (Figs. 3(e) and (f)) and finally collapsed (Fig. 3(g)). After bubble collapse, a new bubble generated, expanded, shrank and collapsed as shown in Figs. 3(h)-(l). This process continued repeatedly. Figure 4 showed a plot of bubble radius measured from the high speed camera as a function of time. It is noted that the radius was measured in horizontal direction. The first bubble had the highest maximum radius of $\sim 3.8 \mathrm{~mm}$ and the longest bubble collapse time of $\sim 530 \mu \mathrm{s}$. The maximum radii and bubble collapse times of the second and third bubbles were 3.7 and $1.8 \mathrm{~mm}$ and 400 and $313 \mu \mathrm{s}$, respectively. One point should be noted that the time intervals between adjacent AEs in Fig. 2 corresponded to the bubble collapse times in Fig. 4. Although there was a time discrepancy of about $5 \mu \mathrm{s}$, which was related to the time resolution of the high speed camera (6.7 $\mu$ s for $150000 \mathrm{fps}$ ), it was reasonable that bubble collapse was the source of the second to forth AEs. The source of the first AE was related to laser ablation. The results shown in Figs. 2-4 were a specific case for high laser energy. Most of experiments detected only two AE waveforms, the previous one from laser ablation and the other from the first bubble collapse.

\subsection{Phenomena during LSP}

According to high speed camera observation, a schematic diagram of phenomena occurred during LSP is constructed and shown in Fig. 5. It is noted that bubbles in this figure are shown in the cross-section views. When the laser with high enough power is irradiated onto the sample surface, the laser ablation occurs and the sample is converted to plasma (Fig. 5(a)). This plasma does not exist in contact with liquid but exists enveloped by vapor generated from the evaporation of the liquid by the heat of the plasma. Namely, the plasma generated a cavitation bubble (Fig. 5(b)). This bubble expands to reduce its internal pressure and finally reaches its maximum size (Fig. 5(c)). At the moment of maximum expansion, the pressure inside the bubble is much lower than the static ambient pressure, so the bubble starts to shrink. In contrast to bubble expansion which the bubble expands spherically, the bubble collapse starts at the top of the bubble which can be seen in the Figs. 3(e) and (f). The top part of bubble seemed to be flat. This phenomenon is related to the water jet (Fig. 5(d)), which generates when the bubble is located near a solid boundary ${ }^{15)}$. This water jet flows toward the bubble center and strongly hits against the sample when the bubble collapse resulting in a secondary shock wave emitted in the surrounding environment (Fig. 5(e)). This shock wave is detected by AE sensor as the secondary waveform. After that the bubble expands again (Fig. 5(f)). The process of bubble expansion and collapse repeats several times until the bubble splits into small bubbles.

It is known that cavitation bubbles change their behavior,

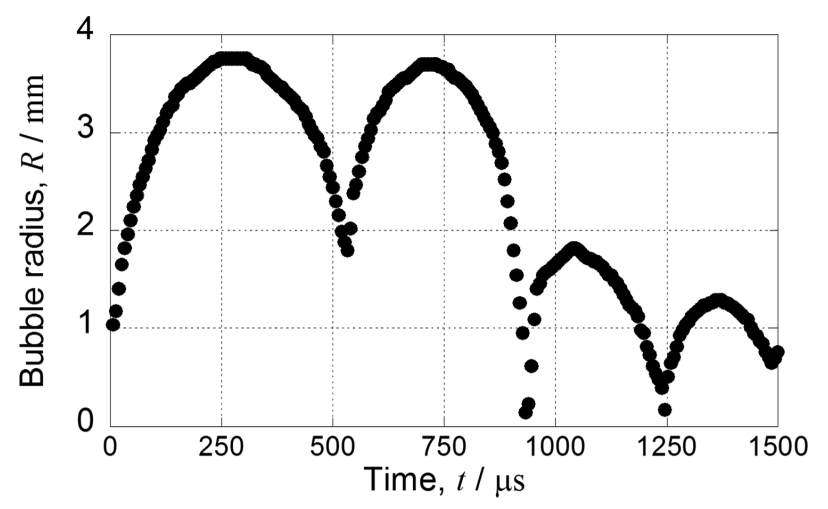

Fig. 4 Example of bubble radius from high speed camera observation at laser power density of $5.0 \mathrm{GW} / \mathrm{cm}^{2}$.
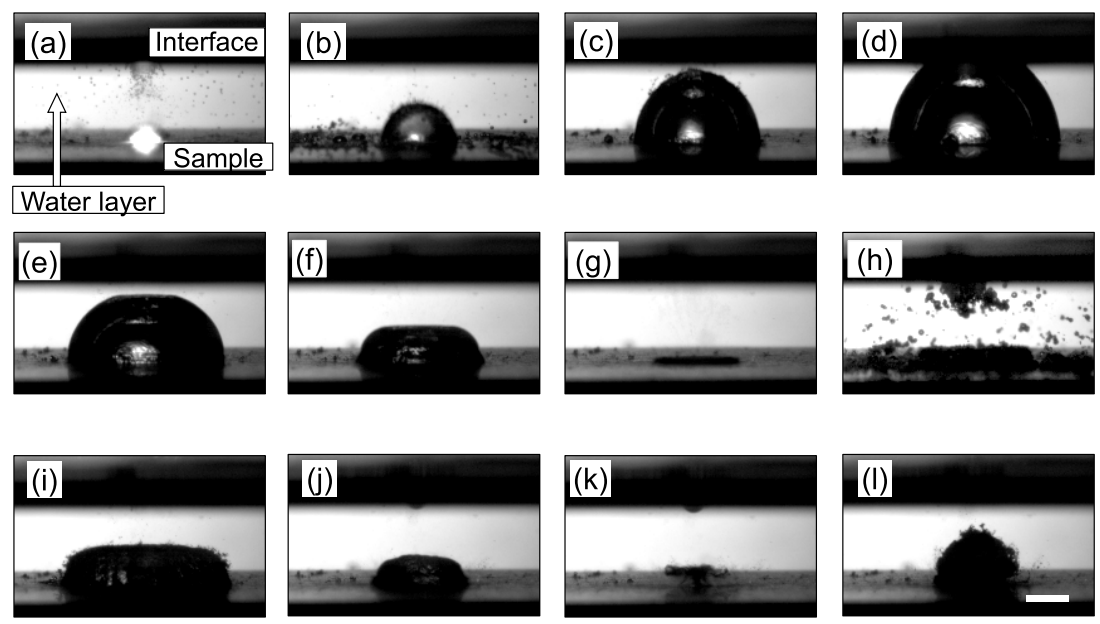

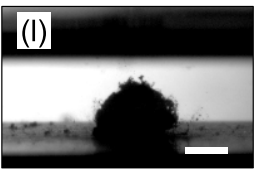

$2 \mathrm{~mm}$

Fig. 3 Observation of sample surface by high speed camera during laser shock peening at laser power density of $5.0 \mathrm{GW} / \mathrm{cm}^{2}$ at (a) $6.7 \mu \mathrm{s}$, (b) $26.6 \mu$ s, (c) $86.6 \mu \mathrm{s}$, (d) $259.8 \mu \mathrm{s}$, (e) $399.7 \mu \mathrm{s}$, (f) $479.6 \mu \mathrm{s}$, (g) $532.9 \mu \mathrm{s}$, (h) $552.9 \mu \mathrm{s}$, (i) $699.5 \mu \mathrm{s}$, (j) $899.3 \mu \mathrm{s}$, (k) $932.6 \mu \mathrm{s}$ and (l) $1045.9 \mu \mathrm{s}$ from laser irradiation. 

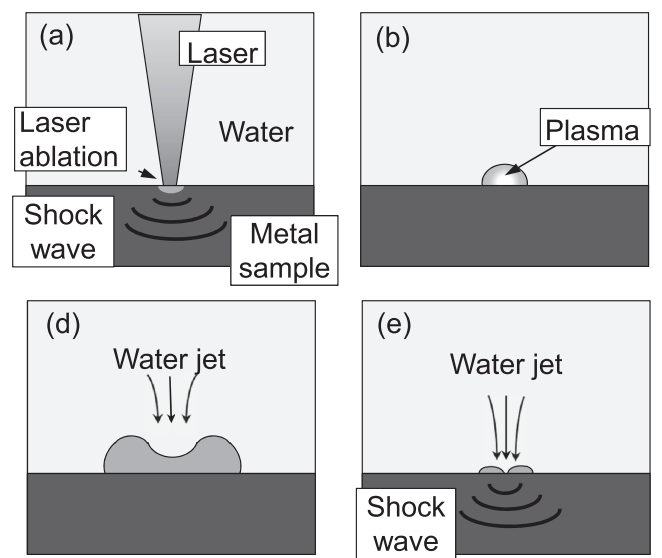
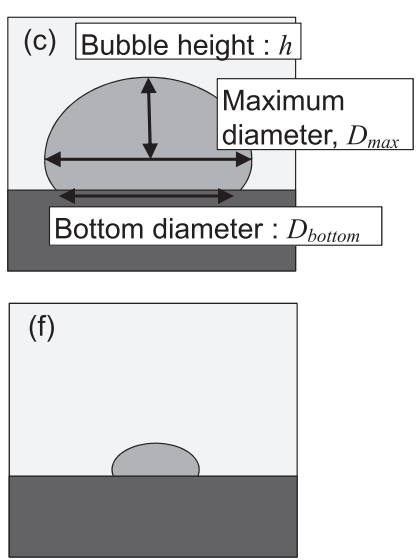

Fig. 5 Schematic sectioned diagrams of cavitation bubble during LSP at (a) laser irradiation, (b) bubble generation, (c) maximum size, (d) shrinking, (e) bubble collapse and (f) rebound.

depending on the dynamic properties of surrounding materials ${ }^{16)}$. For further analysis, several parameters extracted from bubble observation and AE waveforms were used. Figure 5(c) shows parameters extracted from bubble observation at its maximum size. These consisted of bottom diameter $\left(D_{\text {bottom }}\right)$, maximum diameter $\left(D_{\max }\right)$ and bubble height $(h)$. When confinement layer is pure water, $D_{\text {bottom }}$ is equal to $D_{\max }$. The parameters from $\mathrm{AE}$ waveform contained interval time between AEs generated from laser ablation and the first bubble collapse $(\Delta t)$, impact force from laser ablation $\left(I_{L}\right)$, and impact force from first bubble collapse $\left(I_{B}\right)$. Two latter parameters were derived from inverse analysis.

\subsection{Effect of water temperature}

A plot of AE interval time, $\Delta t$, as a function of water temperature is shown in Fig. 6(a). It clearly showed that $\Delta t$ tended to increase with increasing water temperature and power density. The latter one had a pronounced influence. Since AE interval time can be considered as bubble collapse time, the water temperature affected the size and collapse of the cavitation bubble. It is reasonable that the bubble size tended to increase with water temperature because bigger bubble required longer time to collapse.

Temperature affects bubble dynamics in several ways such as altering the viscosity, density, surface tension, etc. With an increase of water temperature, the gap between the inter-molecules increases significantly and the attractive force decreases, which causes decrease in viscosity and surface tension. For example, the dynamic viscosity of the water at $278 \mathrm{~K}$ is $1.52 \mathrm{mPa} \cdot \mathrm{s}$, which is about three times of that at $323 \mathrm{~K}$ $(0.548 \mathrm{mPa} \cdot \mathrm{s})$ as shown in Table 2. Liu et al. ${ }^{16)}$ studied the effect of water temperature on the behavior of a laser-induced cavitation bubble near a rigid boundary and also found that increasing water temperature significantly increased the maximum bubble radius. They mentioned two possible reasons. First, the physical properties such as viscosity and surface tension of high temperature water are quite different from those of normal-temperature water. Second, the vapor pressure rapidly increases as the fluid temperature approaches to the saturation temperature. The latter reasons might affect the pressure generated from bubble collapse.

Figure 6(b) shows a plot of impact forces determined from inverse analysis as a function of water temperature. The data
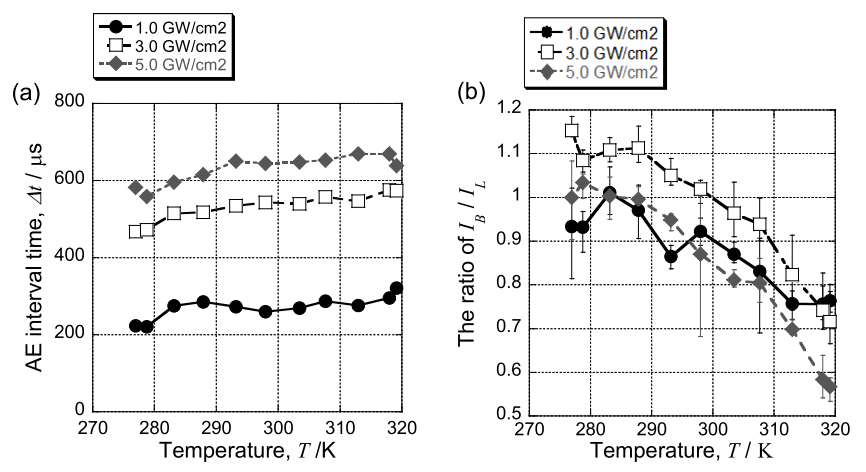

Fig. 6 Effect of water temperature on (a) AE interval time $(\Delta t)$ and (b) the ratio of impacts by laser ablation and bubble collapse $\left(I_{B} / I_{L}\right)$.

were given as a ratio of impact force generated from bubble collapse, $I_{B}$, to that generated from laser irradiation, $I_{L}$. The peak pressure from the laser ablation in confined regime can be estimated by ${ }^{5}$ :

$$
\begin{gathered}
P_{L}=0.01 \sqrt{\frac{\alpha}{2 \alpha+3}} \sqrt{Z_{L}} \sqrt{I} \\
\frac{2}{Z_{L}}=\frac{1}{Z_{S}}+\frac{1}{Z_{C}} \\
Z=\rho \cdot U
\end{gathered}
$$

where $P_{L}(\mathrm{GPa})$ is the peak pressure, $Z\left(\mathrm{~g} / \mathrm{cm}^{2} \mathrm{~s}\right)$ is combined shock impedance defined by the following eq. (3), where $Z_{S}$ and $Z_{C}$ the impedance of the sample and the confinement layer, respectively, $\alpha$ is a constant that is related to efficiency of laser ablation and $\alpha$ will be close to 0.1 if most of laser energy absorbed by material surface and $I\left(\mathrm{GW} / \mathrm{cm}^{2}\right)$ is laser power density. The impedance of the sample and the confinement layer can be calculated from eq. (4), where $\rho\left(\mathrm{g} / \mathrm{cm}^{3}\right)$ is the density of the material and $U(\mathrm{~m} / \mathrm{s})$ is the shock wave velocity within the material which is equal to the sound velocity. Table 2 shows the relationship between temperature and various properties of water. The impedance values of water, which were calculated from eq. (4), increased $\sim 7 \%$ with increasing water temperature from 278 to $323 \mathrm{~K}$. If we assumed that the impedance of the sample was constant within this temperature range, the effect of water temperature on $P_{L}$ was 
Table 2 Relationship between water temperature and density, sound velocity, impedance and saturated vapor pressure of water.

\begin{tabular}{|c|c|c|c|c|c|}
\hline Water temperature/K & $\begin{array}{l}\text { Density } \\
/ \mathrm{g} \cdot \mathrm{cm}^{-3}\end{array}$ & $\begin{array}{l}\text { Sound velocity } \\
\qquad / \mathrm{m} \cdot \mathrm{s}^{-1}\end{array}$ & $\begin{array}{c}\text { Dynamic viscosity } \\
/ 10^{-3} \mathrm{~Pa} \cdot \mathrm{s}\end{array}$ & 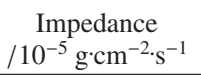 & $\begin{array}{c}\text { Saturated vapor pressure } \\
\qquad / 10^{3} \mathrm{~Pa}\end{array}$ \\
\hline 278 & 0.9998 & 1430 & 1.52 & 1.43 & 0.87 \\
\hline 283 & 0.9997 & 1450 & 1.31 & 1.45 & 1.23 \\
\hline 293 & 0.9982 & 1480 & 1 & 1.48 & 2.34 \\
\hline 303 & 0.9956 & 1510 & 0.797 & 1.5 & 4.24 \\
\hline 313 & 0.9922 & 1530 & 0.653 & 1.52 & 7.38 \\
\hline 323 & 0.988 & 1540 & 0.548 & 1.52 & 12.34 \\
\hline
\end{tabular}

only $\sim 3 \%$ according to eq. (2). Therefore, it could infer that the effect of water temperature on impact force from laser ablation was small and the value of $I_{L}$ for a fixed laser power density could be estimated as a constant value within 278 to $323 \mathrm{~K}$. Figure 6(b) shows that although there were scatterings in data, the ratio of $I_{B} / I_{L}$ seemed to be constant up to $\sim 288 \mathrm{~K}$ and then decreased with increasing water temperature. In the case of power densities of 1.0 and $5.0 \mathrm{GW} / \mathrm{cm}^{2}, I_{B}$ were likely lower than $I_{L}$ within the test temperature range while the opposite trend was observed for a power density of $3.0 \mathrm{GW} /$ $\mathrm{cm}^{2}$ up to $298 \mathrm{~K}$. A possible explanation for the decrease of $I_{B}$ with increasing of water temperature is as following. The vapor pressure inside the bubble can be considered as saturation vapor pressure, $P_{S}$, which is a function of temperature and can be expressed by Tetens's formula ${ }^{17)}$ as

$$
P_{S}=0.611 \times 10^{\left(\frac{7.5 T}{T+237.3}\right)}
$$

where $T$ is water temperature in degree Celsius and $P_{S}$ is in $\mathrm{kPa}$. According to this equation, $P_{S}$ increase with increasing temperature as shown in Table 2. Since this pressure was equal to the vapor pressure inside the bubble, the increase of the pressure with water temperature became the resistance of water jet during the water collapse and reduced the magnitude of shock wave. One point should be noted that the increase of the $P_{S}$ with temperature was an exponential manner, so the resistance of water jet was not significant in low temperature range where the $P_{S}$ values were not very different. This behavior was in good agreement with the findings in Fig. 6(b). The ratio of $I_{B} / I_{L}$ kept constant within 278 to $288 \mathrm{~K}$, which the $P_{S}$ values within this temperature range changed a little. Therefore, it is reasonable that the decrease of $I_{B}$ with increasing water temperature was related to an increase of saturation water temperature.

\subsection{Effect of water thickness}

Examples of detected AE waveforms and high speed camera observation of some experimental conditions are shown in Figs. 7 and 8, respectively. In the case of 4-mm water thickness and $3.0-\mathrm{GW} / \mathrm{cm}^{2}$ laser power density, the results are shown in Figs. 7(a) and 8(a). Two AE waveforms, which were generated from laser ablation and bubble collapse, were detected at 0 and $367 \mu \mathrm{s}$, respectively. The high speed camera observation showed that the bubble generated at $13 \mu \mathrm{s}$, expanded to its maximum size of $5.6 \mathrm{~mm}$ at $204 \mu$ s and finally collapsed at $373 \mu \mathrm{s}$. The observed phenomena were a typical case as discussed in the section 3.2. Many small cavitation bubbles were observed immediately after laser ablation and bubble collapse (Fig. 8(a) of 13 and $386 \mu$ s). These bubbles were generated by pressure difference after passing shock
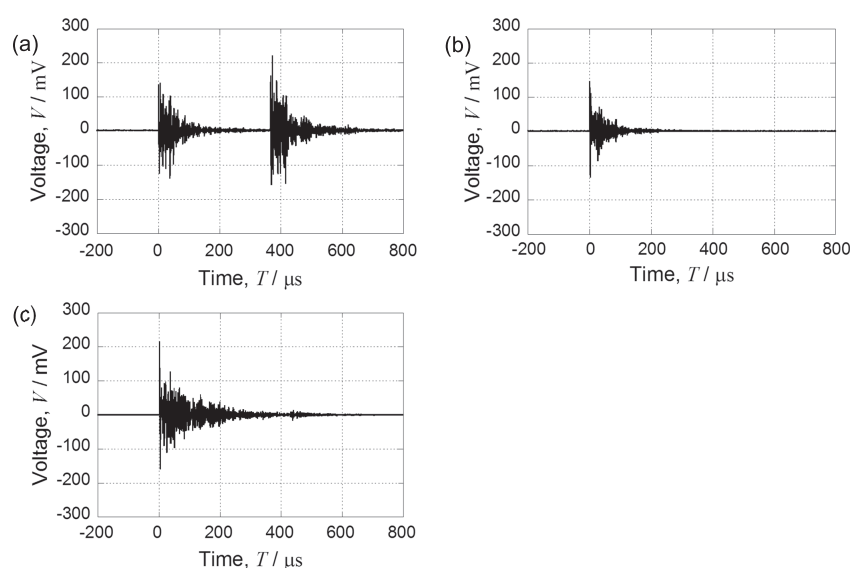

Fig. 7 Detected AE waveforms in cases of (a) water layer of $4 \mathrm{~mm}$, laser power density of $3.0 \mathrm{GW} / \mathrm{cm}^{2}$, (b) water layer of $1 \mathrm{~mm}$, laser power density of $3.0 \mathrm{GW} / \mathrm{cm}^{2}$ and (c) water layer of $5 \mathrm{~mm}$, laser power density of $5.0 \mathrm{GW} / \mathrm{cm}^{2}$.

waves by laser ablation and bubble collapse ${ }^{18)}$. When the water thickness was reduced to $1 \mathrm{~mm}$ with the same laser energy, only AE from laser ablation was detected as shown in Fig. 7(b). The high speed camera observation (Fig. 8(b)) shows that the bubble expanded its size with its upper part contacted to water surface just after $13 \mu$ s from bubble generation. Water splash was also observed as the black color in air layer. This disk-like bubble expanded to its maximum size with a diameter of $7.1 \mathrm{~mm}$ at approximately $1000 \mu$ s and could maintain its shape without collapse up to at least $10000 \mu \mathrm{s}$. Finally, the bubble became smaller and disappeared from the water surface. The way of bubble collapse of this bubble was quite different from that observed in typical case, which the bubble shrank immediately after reaching its maximum size and the bubble collapse took place at the sample surface. This finding could be explained as following. If the bubble in this case of 1-mm-thick water layer expanded in hemisphere manner, the top of the bubble should be observed in the air. But the high speed camera showed the disk-like bubble, this meant that the bubble merged with air layer by blowing off water. This phenomenon resulted in a change of pressure inside the bubble which became equal to air pressure and hence the pressure difference between inside and outside the bubble was much small. Therefore, the bubble could keep its shape and resulted in a long bubble collapse time. Only AE generated by laser ablation was also detected in another case when the laser energy was very high with a sufficient thickness of water layer as shown in Fig. 7(c). The high speed camera observation (Fig. 8(c)) shows that the bubble generation occurred not only at the sample surface but also at the 
(a) Interface
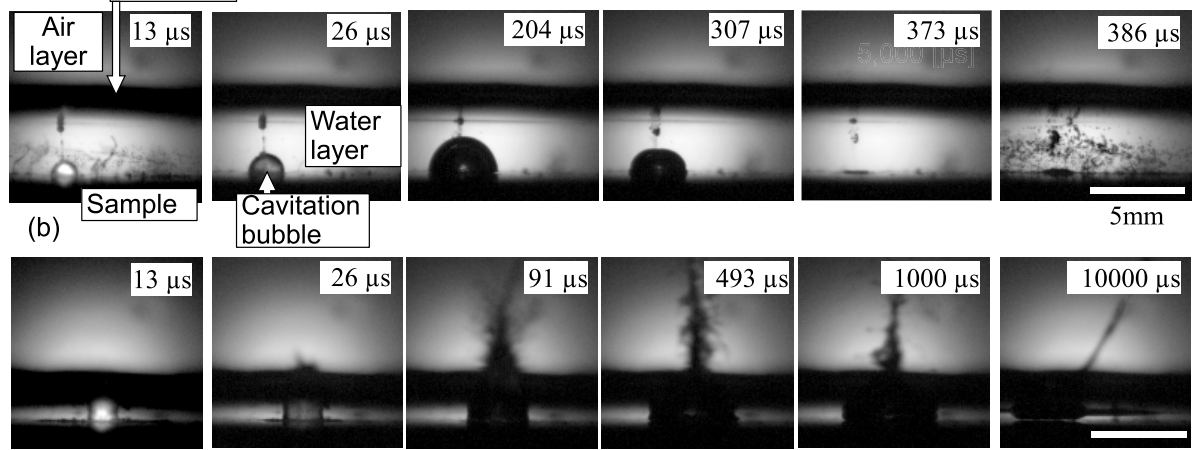

bubble

(c)
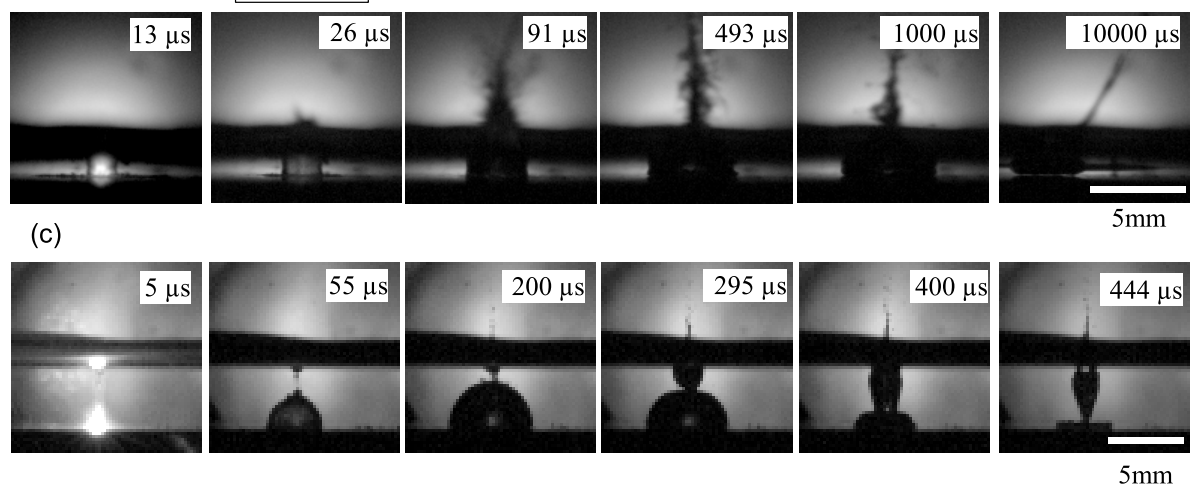

Fig. 8 Observation of sample surface by high speed camera in cases of (a) water layer of $4 \mathrm{~mm}$, laser power density of $3.0 \mathrm{GW} / \mathrm{cm}^{2},(\mathrm{~b}) \mathrm{water}$ layer of $1 \mathrm{~mm}$, laser power density of $3.0 \mathrm{GW} / \mathrm{cm}^{2}$ and (c) water layer of $5 \mathrm{~mm}$, laser power density of $5.0 \mathrm{GW} / \mathrm{cm}^{2}$.

interface of water and air layers. However, the bubble expansion rate of the latter one was quite slow (see Fig. 8(c) at 55 and $200 \mu \mathrm{s}$ ). Two bubbles were coalesced at approximately $300 \mu$ s and then the shape of the bubble changed into a unique manner. This coalescence of the bubbles seemed to prevent the water jet generation and therefore no shock wave was generated. According to these findings, it could be inferred that AE method is a powerful tool to monitor LSP process. Any irregular phenomenon from bubble collapse can be notified by no second AE waveform.

A plot of impact forces obtained by deconvolution technique of detected $\mathrm{AE}$ waveforms at laser power density of $3.0 \mathrm{GW} / \mathrm{cm}^{2}$ as a function of water thickness is shown in Fig. 9. In the case of 1-mm water thickness, $I_{B}$ could not be determined because there was no bubble collapse on the sample surface as mentioned above. When the water thickness was $2 \mathrm{~mm}, I_{B}$ values were lower than $I_{L}$. This behavior might be related to the confinement of plasma and/or bubble collapse behavior. Since plasma was confined by the weight of confinement layer on sample surface, the confinement of plasma might be imperfection when the water layer was not thick enough. When the water thickness was higher than $3 \mathrm{~mm}$, although there were scattering in data both $I_{L}$ and $I_{B}$ tended to decrease with increasing water thickness and the values of $I_{B}$ were higher than those of $I_{L}$. These findings were related to the effects of air pressure and the interaction between bubble and the interface of air and water layer. A summation of $I_{L}$ and $I_{B}$ was also given in Fig. 9. If the magnitude of residual stress by peening is directly related to the magnitude of total force applied on the surface, it can infer that the water thickness of 3 to $5 \mathrm{~mm}$ yielded a good peening effect. This finding shows that AE is a powerful tool for process optimization.

\subsection{Effect of viscosity of confinement layer}

In the previous section, it has shown that not only viscosity of water but also saturation vapor pressure changed with temperature and consequently affected the bubble collapse be-

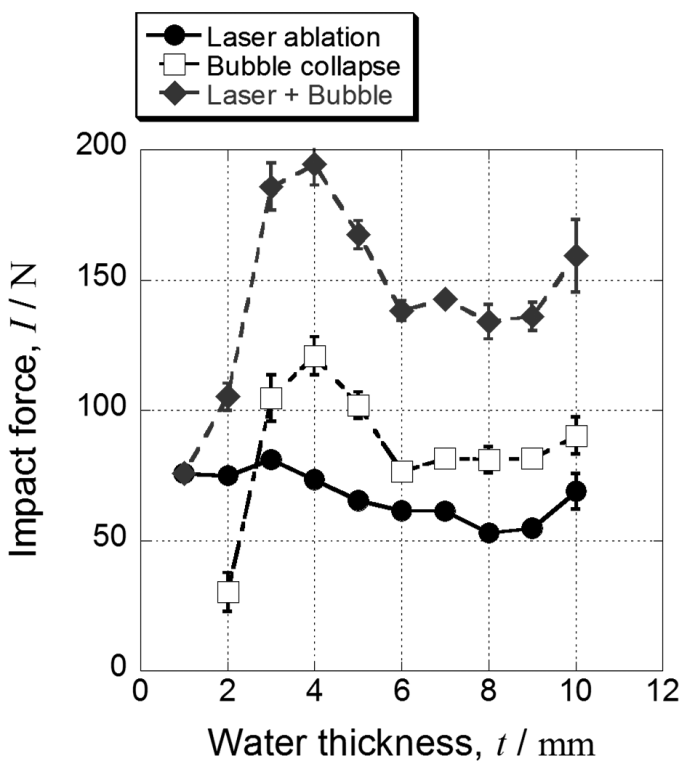

Fig. 9 Effect of water thickness on impacts by laser ablation and bubble collapse at laser power density of $3.0 \mathrm{GW} / \mathrm{cm}^{2}$.

havior. In this section, a mixture of glycerin and water was used as the confinement layer in order to investigate the effect of viscosity. Table 1 shows the viscosity of confinement layer obtained from the calculation of literature data ${ }^{11)}$.

Figure 10 shows sequential images recorded by the high speed camera during LSP with a laser power density of $5.0 \mathrm{GW} / \mathrm{cm}^{2}$ and a confinement layer of pure glycerin. It was clear that the bubble expansion and collapse in glycerin were different from those observed in pure water. While the bubble expanded in hemisphere manner in water (see Figs. 3(a)-(d)), the shape of bubble in glycerin was likely ellipsoid (see Figs. 10(a)-(d)). The bubble expanded up to its maximum size (Fig. 10(d)) and then became smaller. Since the bubble wall could move faster in the glycerin than at the sample surface, the bubble shrank in the unique manner like a cone shape (Figs. 10(f)-(g)). Finally, the bubble collapsed 

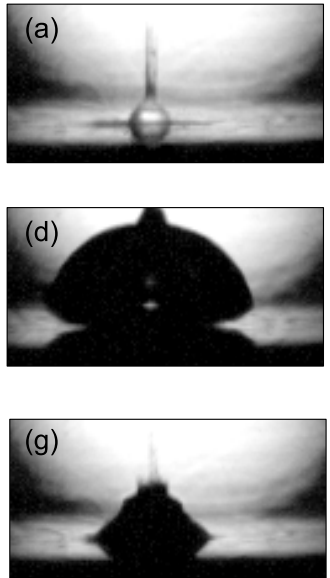
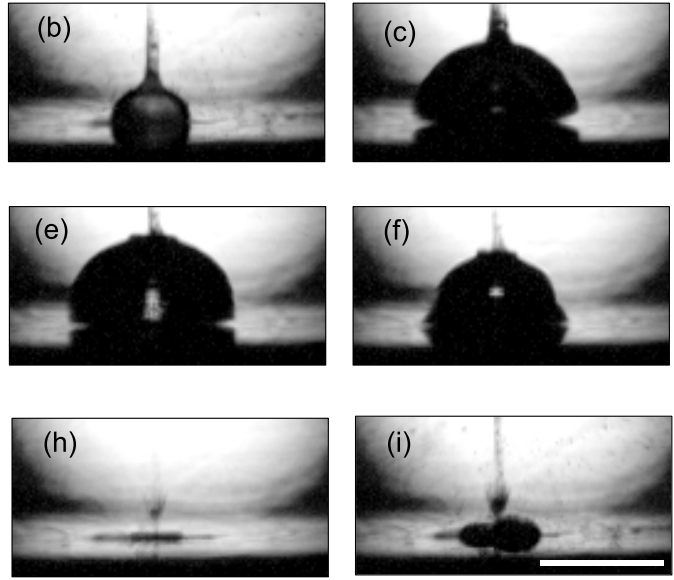

$5 \mathrm{~mm}$

Fig. 10 Observation by high speed camera during laser shock peening in glycerin at laser power density of $5.0 \mathrm{GW} / \mathrm{cm}^{2}$ at (a) $2.6 \mu \mathrm{s}$, (b) $16.9 \mu \mathrm{s}$, (c) $109.8 \mu \mathrm{s}$, (d) $302.6 \mu \mathrm{s}$, (e) $481.2 \mu \mathrm{s}$, (f) $545.5 \mu \mathrm{s}$, (g) $588.3 \mu \mathrm{s}$, (h) $616.9 \mu \mathrm{s}$ and (i) $631.2 \mu \mathrm{s}$ from laser irradiation.
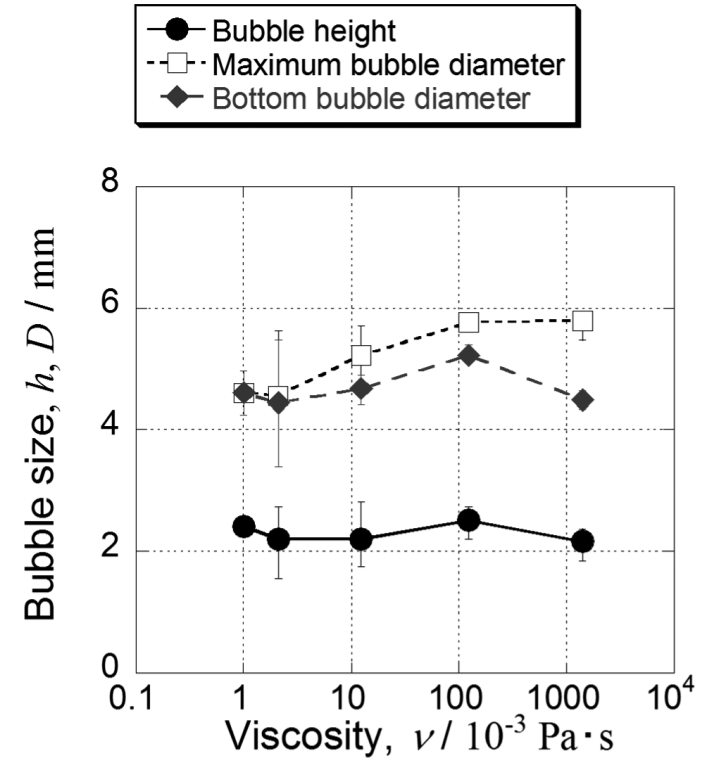

Fig. 11 Effect of the viscosity of the confinement layer on bubble about maximum diameter $\left(D_{\max }\right)$ and bottom diameter $\left(D_{\text {bottom }}\right)$ and bubble height $(h)$ at laser power density of $3.0 \mathrm{GW} / \mathrm{cm}^{2}$.

(Fig. 10(h)) and rebounded (Fig. 10(i)). Various parameters of bubble as shown in Fig. 5(c) from several experimental conditions were derived and further analyzed. A plot of bubble height $(h)$, maximum diameter $\left(D_{\max }\right)$ and bottom diameter $\left(D_{\text {bottom }}\right)$ as a function of viscosity $(v)$ for a laser power density of $3.0 \mathrm{GW} / \mathrm{cm}^{2}$ is shown in Fig. 11 . Both $D_{\max }$ and $D_{\text {bottom }}$ seemed to increase with increasing viscosity, while no significant change in bubble height was observed. Similar trends were also observed in other LSP conditions with different power energies. Since the shape of the bubble tended to change from hemisphere to ellipsoid with increasing viscosity, ratios of $D_{\text {bottom }}$ to $D_{\max }$ was plotted with viscosity to clarify its effect and shown in Fig. 12. It can be seen that although there were scatterings of data, $D_{\text {bottom }} / D_{\max }$ decreased as the viscosity increased for all the three laser powers. The $D_{\text {bottom }} /$ $D_{\max }$ showed a sudden decrease up to $10-\mathrm{mPa}$ viscosity, and then maintained its value up to $\sim 100-\mathrm{mPa} \cdot$ viscosity and finally decreased with increasing viscosity. The AE interval time was also plotted in Fig. 12 and it tended to increase with in-

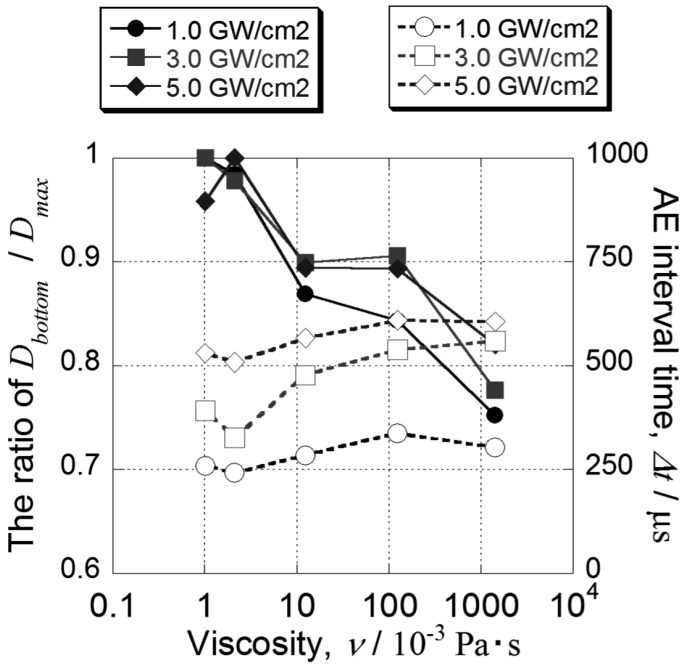

Fig. 12 Effect of viscosity of confinement layer on the ratio of $D_{\text {bottom }} / D_{\max }$ and $\mathrm{AE}$ interval time $(\Delta t)$

creasing viscosity and laser power. Since the AE interval time could be considered as the bubble collapse time, this meant that bubble required longer time to collapse in liquid with higher viscosity. This behavior might be related to a decrease of bubble wall velocity with viscosity and a change of bubble collapse phenomena.

A relationship between impact forces by laser ablation $\left(I_{L}\right)$ and bubble collapse $\left(I_{B}\right)$ and viscosity is given in Fig. 13(a). In the case of $1.0-\mathrm{GW} / \mathrm{cm}^{2}$ laser power density, both $I_{L}$ and $I_{B}$ tended to increase with increasing viscosity, although not significantly. The effect of viscosity on $I_{L}$ became pronounced with higher laser powers. The values of $I_{L}$ seemed to be higher than those of $I_{B}$ for 3.0 - and $5.0-\mathrm{GW} / \mathrm{cm}^{2}$ laser power densities. The $I_{B}$ of $3.0-\mathrm{GW} / \mathrm{cm}^{2}$ laser power density slightly increased with viscosity while the opposite trend was observed for $5.0-\mathrm{GW} / \mathrm{cm}^{2}$ laser power density. The increase of $I_{L}$ with viscosity can be explained as follows. According to eq. (2), the peak pressure from the laser ablation increases with the shock impedance, which can be calculated from eqs. (3) and (4). The density and sound velocity of the confinement layer used in present study increased with increasing glycerin volume because glycerin has higher density $\left(1.26 \mathrm{~g} / \mathrm{cm}^{3}\right)$ and 

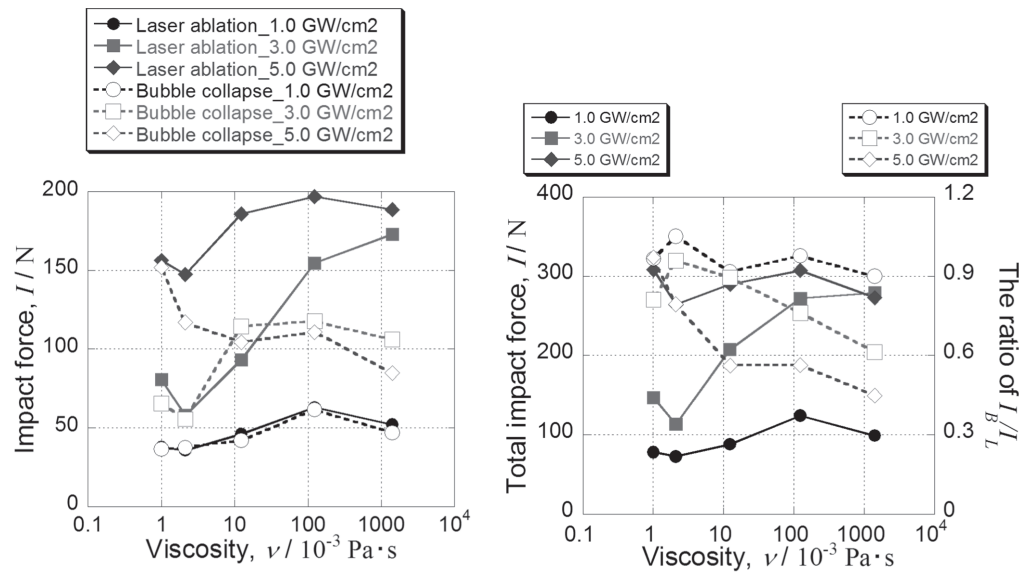

Fig. 13 Effect of the viscosity on (a) average values of impacts by laser ablation and bubble collapse and (b) summation value of $I_{B}$ and $I_{L}$, and the ratio of $I_{B} / I_{L}$, at various laser power densities.

faster sound velocity $(1980 \mathrm{~m} / \mathrm{s}$ at $293 \mathrm{~K})$ than those of water. Therefore, the impedance of the confinement layer increased according to eq. (4) and resulted in an increase of the shock impedance. It should be noted that the peak pressure from the laser ablation is also in proportion to the square root of laser power density. This might explain a small effect of viscosity on $I_{L}$ in the case of $1.0-\mathrm{GW} / \mathrm{cm}^{2}$ laser power density. Consider $I_{B}$ which demonstrates the magnitude of liquid jet impact, it is known that viscosity has a slowing effect on the dynamic processes of bubble expansion and collapse. The viscosity of the confinement layer would exert on the bubble radial force opposite to the attempted motion in the layer, dissipating the bubble's energy and retarding the rapid collapse of a cavity bubble. This behavior could be confirmed with an increase of AE interval time in Fig. 12. When the viscosity is larger, higher viscous resistance on the bubble is expected and resulted in a decrease of liquid-jet impact. The present results obtained with $5.0-\mathrm{GW} / \mathrm{cm}^{2}$ laser power density showed such a behavior. However, $I_{B}$ of $1.0-$ and $3.0-\mathrm{GW} /$ $\mathrm{cm}^{2}$ laser power density slightly increased with viscosity. This behavior was unexpected and might be related to the complex interaction of liquid-jet and bubble. Liu et al. ${ }^{19)}$ measured the liquid-jet impact force when the bubble collapsed near a rigid body in a mixture of glycerin and water of various concentrations using a fiber-coupling optical beam deflection (OBD). They found that the liquid-jet impact force decreased sharply with increasing viscosity which was the same for the result of $5.0-\mathrm{GW} / \mathrm{cm}^{2}$ laser power density in the present study.

A ratio of $I_{B}$ to $I_{L}$ and a summation of $I_{L}$ and $I_{B}$ were plotted as a function of viscosity and shown in Fig. 13(b). The $I_{B} / I_{L}$ tended to decrease with viscosity and the decreasing degree was more pronounced with increasing laser power. This behavior inferred that the effect of a cavitation bubble on LSP could be controlled by adjusting viscosity and laser power. The total value of impact forces $\left(I_{L}+I_{B}\right)$ tended to increase with viscosity, although not significantly for 1.0 and $5.0-\mathrm{GW} /$ $\mathrm{cm}^{2}$. This plot might be a useful hint to design/optimize LSP condition.

\section{Conclusions}

The effect of confinement layer during LSP was quantita- tively evaluated by AE analysis and high speed camera. The results obtained from this research are as follows:

(1) Impact force by laser ablation was affected little by water temperature ranged between 278 and $323 \mathrm{~K}$. On the other hand, impact force by a cavitation bubble collapse decreased with increasing water temperature.

(2) When water thickness was thin or laser energy was very high, no impact by bubble collapse emit because a cavitation bubble touch air layer till the bubble collapse. Impact force by bubble collapse showed the maximum value when thickness of water layer was 3-5 mm.

(3) Impact force by laser ablation increased with increasing viscosity of confinement layer. On the other hand, the opposite trend was observed in impact force by bubble collapse because of a change of bubble collapse behavior.

\section{REFERENCES}

1) T. J. Rockstroh: Proc. ICALEO (2005) pp. 703-709.

2) Y. Sano, K. Akita, K. Masaki and Y. Ochi: J. Laser Micro Nanoen. 1 (2006) 161-166.

3) L. Berthe, R. Fabbro, P. Peyre, L. Tollier and E. Bartnicki: J. Appl. Phys. 82 (1997) 2826-2832.

4) X. Hong, S. Wang, D. Guo, H. Wu, J. Wang, Y. Dai, X. Xia and Y. Xie: Opt. Lasers Eng. 29 (1998) 447-455.

5) R. Fabbro, J. Fournier, P. Ballard, D. Devaux and J. Virmont: J. Appl. Phys. 68 (1990) 775-784.

6) C.Y. Cui, Y.J. Hong, J.F. Ye, M. Wen and N.L. Li: Appl. Phys., A Mater. Sci. Process. 103 (2011) 239-243.

7) M. Gelfi, E. Bontempi, R. Roberti and L.E. Depero: Acta Mater. 52 (2004) 583-589.

8) L.M. Barker and R.E. Hollenbach: J. Appl. Phys. 43 (1972) 4669-4675.

9) M. Takemoto, M. Nakamura, S. Masano and S. Ueno: J. Acous. Emiss. 27 (2009) 241-253.

10) T. Takata, M. Enoki, P. Chivavibul, A. Matsui and Y. Kobayashi: Mater. Trans. 57 (2016) 674-680.

11) J.B. Segur and H.E. Oberstar: Ind. Eng. Chem. 43 (1951) 2117-2120.

12) K. Ito and M. Enoki: Mater. Trans. 48 (2007) 1221-1226.

13) M.G.R. Sause: J. Acous. Emiss. 29 (2011) 184-196.

14) D.J. Buttle and C.B. Scruby: Wear 137 (1990) 63-90.

15) A. Philipp and W. Lauterborn: J. Fluid Mech. 361 (1998) 75-116.

16) X.-M. Liu, X.-H. Liu, Y.-F. Hou, J. He, J. Lu and X.-W. Ni: Lasers Eng. 21 (2011) 181-193.

17) O. Tetens: Z. Geophys. 6 (1930) 297-309.

18) C.D. Ohl: Phys. Fluids 14 (2002) 3512-3521.

19) X-M. Liu, J. He, J. Lu and X-W. Ni: Jpn. J. Appl. Phys. 48 (2009) 016504 . 